\title{
A PAIR OF BIORTHOGONAL POLYNOMIALS FOR THE SZEGÖ-HERMITE WEIGHT FUNCTION
}

\author{
N.K. THAKARE \\ Mathematics Department \\ University of Poona \\ Pune - 411007 , INDIA \\ and
}

\section{M.C. MADHEKAR}

Milind College of Science Aurangabad - 431 004, INDIA

(Received April 1, 1987 and in revised form August 28, 1987)

ABSTRACT. A pair of polynomial sequences $\left\{S_{n}^{\mu}(x ; k)\right\}$ and $\left\{T_{m}^{\mu}(x ; k)\right\}$ where $S_{n}^{\mu}(x ; k)$ is of degree $n$ in $x^{k}$ and $T_{m}^{\mu}(x ; k)$ is of degree $m$ in $x$, is constructed. It is shown that this pair is biorthogonal with respect to the Szegö-Hermite weight function $|x|^{2 \mu} \exp \left(-x^{2}\right),(\mu>-1 / 2)$ over the interval $(-\infty, \infty)$ in the sense that

$$
\begin{aligned}
\int_{-\infty}^{\infty}|x|^{2 \mu} \exp \left(-x^{2}\right) S_{n}^{\mu}(x ; k) T_{m}^{\mu}(x ; k) d x & =0, \text { if } m \neq n \\
& \neq 0, \text { if } m=n
\end{aligned}
$$

where $m, n=0,1,2, \ldots$ and $k$ is an odd positive integer.

Generating functions, mixed recurrence relations for both these sets are obtained. For $k=1$, both the above sets get reduced to the orthogonal polynomials introduced by professor Szegö.

KEYS WORDS AND PHRASES. Szego-Hermite weight function, Biorthogonal pair, Generating functions, Recurrence relations, etc.

1980 AMS SUBJECT CLASSIFICATION CODE. 33A65, 33A99, 42C05, $42 \mathrm{C} 99$.

\section{INTRODUCTION.}

The biorthogonality conditions are useful in the computations involving the penetration of gamma rays through matter as well as in determining the moments of a hypergeometric distribution function. The notion of biorthogonality dates back to Didon [1] and Deruyts [2]. The questions of constructing biorthogonal pairs of polynomials corresponding to the weight functions of classical orthogonal polynomials were taken up by Konhauser [3] for the Laguerre weight function $\mathrm{x}^{\alpha} \mathrm{e}^{-\mathrm{x}}$, by Toscano [4], Chai [5], Carlitz [6] and Madhekar and Thakare [7] for the Jacobi weight function $(1-x)^{\alpha}(1+x)^{\beta}$ and by Thakare and Madhekar [4] for the Hermite weight function $\exp \left(-x^{2}\right)$. The Szegö-Hermite polynomials $H_{n}^{\mu}(x)$ are orthogonal w.r.t. the Szegö-Hermite weight function $|x|^{2 \mu} \exp \left(-x^{2}\right),(\mu>-1 / 2)$ over the interval $(-\infty, \infty)$ and these are found 
useful in connection with Gauss-Jacobi mechanical quadrature, see Szegö [8]. For $\mu=0$, Szegö-Hermite polynomials are just the classical Hermite polynomials.

2. A BIORTHOGONAL SYSTEM.

We shall construct a pair of biorthogonal polynomials w.r.t. the Szego-Hermite weight function $|x|^{2 \mu} \exp \left(-x^{2}\right), \mu>-1 / 2$. Consider the following pair of polynomial sequences.

$$
\begin{aligned}
& s_{n}^{\mu}(x ; k)=2^{n} \Gamma((k n+k-k \varepsilon) / 2+\mu+\varepsilon) \\
& \text { - } \sum_{j=0}^{[1 / 2]}(-1)^{j}\left(\begin{array}{c}
{[n / 2]} \\
j
\end{array}\right) x^{n k-2 k j} / \Gamma((k n+1+\varepsilon) / 2-k j+\mu) . \\
& T_{n}^{\mu}(x ; k)=(-1)^{[n / 2]} 2^{n} \sum_{r=0}^{[n / 2]} x^{n-2 r} /([n / 2]-r) ! \quad \sum_{s=0}^{[n / 2]-r}(-1)^{s}\left(\begin{array}{c}
{[n / 2]-r} \\
s
\end{array}\right) \\
& \text { - }((2 \mathrm{~s}+(\mathrm{k}+1) \varepsilon+2 \mu+1) / 2 \mathrm{k})_{[\mathrm{n} / 2]} \text {, }
\end{aligned}
$$

where the value of $\varepsilon$ is 0 or 1 according to even or odd nature of $n$. Throughout this paper $\varepsilon$ always has this meaning; and [p] is the greatest integer less than or equal to $\mathrm{p}$.

It is fairly easy to verify after reverting the order of summation for even and odd integers that

$$
\begin{aligned}
& s_{2 n}^{\mu}(x ; k)=(-1)^{n} 2^{2 n} \Gamma(k n+\mu+k / 2) \sum_{j=0}^{n}(-1)^{j}\left(\begin{array}{l}
n \\
j
\end{array}\right) x^{2 k j} / \Gamma(k j+\mu+1 / 2), \\
& =(-1)^{n} 2^{2 n} n ![\Gamma(k n+\mu+k / 2) / \Gamma(k n+\mu+1 / 2)] z_{n}^{\mu-1 / 2}\left(x^{2} ; k\right) ; \\
& s_{2 n+1}^{\mu}(x ; k)=(-1)^{n} 2^{2 n+1} \Gamma(k n+\mu+1+k / 2) \sum_{j=0}^{n}(-1)^{j}\left(\begin{array}{l}
n \\
j
\end{array}\right) \frac{x^{2 k j+k}}{\Gamma(k j+\mu+1+k / 2)} \\
& =(-1)^{n} 2^{2 n+1} n ! x^{k} z_{n}^{\mu+k / 2}\left(x^{2} ; k\right) ; \\
& \mathrm{T}_{2 \mathrm{n}}^{\mu}(\mathrm{x} ; \mathrm{k})=(-1)^{\mathrm{n}} 2^{2 \mathrm{n}} \sum_{\mathrm{r}=0}^{\mathrm{n}} \frac{2^{2 \mathrm{r}}}{\mathrm{r} !} \sum_{\mathrm{s}=0}^{\mathrm{r}}(-1)^{\mathrm{s}}\left(\begin{array}{l}
\mathrm{r} \\
\mathrm{s}
\end{array}\right)((\mathrm{s}+\mu+1 / 2) / \mathrm{k})_{\mathrm{n}}, \\
& =(-1)^{n} 2^{2 n} n ! \quad Y_{n}^{\mu-1 / 2}\left(x^{2} ; k\right) \\
& T_{2 n+1}^{\mu}(x ; k)=(-1)^{n} 2^{2 n+1} \sum_{r=0}^{n}\left(x^{2 r+1} / r !\right) \sum_{s=0}^{r}(-1)^{s}\left(\begin{array}{l}
r \\
s
\end{array}\right)((s+\mu+1+k / 2) / k)_{n}, \\
& =(-1)^{n_{2} 2 n+1} n ! \times Y_{n}^{\mu+k / 2}\left(x^{2} ; k\right) \text {. }
\end{aligned}
$$

Here $Z_{n}^{\alpha}(x ; k)$ and $Y_{n}^{\alpha}(x ; k)$ is a pair of Konhauser [3] biorthogonal polynomials w.r.t. the Laguerre weight function $x^{\alpha} \exp (-x)$ over $(0, \infty)$ and are given by

$$
z_{n}^{\alpha}(x ; k)=\frac{\Gamma(k n+\alpha+1)}{n !} \quad \sum_{j=0}^{n}(-1)^{j}\left(\begin{array}{l}
n \\
j
\end{array}\right) \frac{x^{k j}}{\Gamma(k j+\alpha+1)}
$$




$$
\mathrm{Y}_{\mathrm{n}}^{\alpha}(\mathrm{x} ; \mathrm{k})=\frac{1}{\mathrm{n} !} \sum_{\mathrm{r}=0}^{\mathrm{n}} \frac{\mathrm{x}^{\mathrm{r}}}{\mathrm{r} !} \sum_{\mathrm{s}=0}^{\mathrm{r}}(-1)^{\mathrm{s}}\left(\begin{array}{l}
\mathrm{r} \\
\mathrm{s}
\end{array}\right)\left((\mathrm{s}+\alpha+1) / \mathrm{k}_{\mathrm{n}}\right) \text {; see Carlitz [9] }
$$

where $\alpha>-1$, and $\mathrm{k}$ is a postive integer, and

$$
\int_{0}^{\infty} x^{\alpha} e^{-x} z_{n}(x ; k) y_{m}^{\alpha}(x ; k) d x=\frac{\Gamma(k n+\alpha+1)}{n !} \delta(n, m) \text { with } \delta(n, m)
$$

the Kronecker's delta. Using [10] one readily obtains the following biorthogonality condition for the sets $\left\{S_{n}^{\mu}(x ; k)\right\}$ and $\left\{T_{m}^{\mu}(x ; k)\right\}$ :

$$
\begin{aligned}
& \int_{-\infty}^{\infty}|x|^{2 \mu} \exp \left(-x^{2}\right) S_{n}^{\mu}(x ; k) T_{m}^{\mu}(x ; k) d x \\
= & 2^{2 n}[n / 2] ! \Gamma(\mu+\varepsilon+(k n+k-k \varepsilon) / 2) \quad \delta(n, m) .
\end{aligned}
$$

An independent proof of $(2.10)$ is also possible by using the Identity of Carlitz [9, p. 249]:

$$
\left.(-j)_{m}=\sum_{r=0}^{m}\left(\begin{array}{c}
k j+c+m-r \\
m-r
\end{array}\right) \underset{s=0}{m-r}(-1)^{s} \underset{s}{m-r}\right)((s+c+1) / k)_{m} .
$$

One has to note, however, that $k$ is involved in $S_{n}^{\mu}(x ; k)$ and $T_{m}^{\mu}(x ; k)$ must be an odd positive integer in view of the existence theorem for biorthogonality due to

Konhauser [10, p.255].

One readily obtains

$$
\begin{aligned}
& \Gamma(k n+k+\mu+1 / 2) S_{2 n+1}^{\mu}(x ; k)=2 x^{k} \Gamma(k n+\mu+1+k / 2) S_{2 n}^{\mu+(k+1) / 2}(x ; k) \text {, and } \\
& T_{\angle n+1}^{\mu}(x ; k)=2 x T_{2 n}^{\mu+(k+1) / 2}(x ; k) \\
& D S_{2 n}^{\mu}(x ; k)=4 n k x^{k-1} \frac{\Gamma(k n+\mu+k / 2)}{\Gamma(k n+\mu+1 / 2)} S_{2 n-1}^{\mu+(k-1) / 2}(x ; k)
\end{aligned}
$$

\section{SOME PROPERTIES.}

Using the relationship (2.3) to (2.6) it is fairly easy to obtain many results for the Szegö-Hermite biorthogonal pair of polynomials from the known results for the Konhauser biorthogonal sets. The results stated below could also be proved directly. Recall the Calvez and Ge'nin [11] generating function in the form (see also Srivastava [12]):

$$
\sum_{n=0}^{\infty}\left(\begin{array}{c}
m+n \\
n
\end{array}\right) Y_{m+n}^{\alpha}(x ; k) t^{n}=R^{(1+\alpha+m k)} \exp \{x(1-R)\} Y_{m}^{\alpha}(x R ; k),
$$

where $m$ is any integer $\geqq 0$ and $R=(1-t)^{-1 / k}$. By handling even and odd cases separately, from (2.5) and (2.6) respectively, one obtains

$$
\begin{aligned}
& \sum_{n=0} T_{2 m+n}^{\mu}(x ; k) t^{n} /[n / 2] ! \\
= & V U^{(\mu+m k+(1+k) / 2)}\left[U^{-k} T_{2 m}^{\mu}(x U ; k)+t T_{2 m+1}^{\mu}(x U ; k)\right] \text { where } U=\left(1+4 t^{2}\right)^{-1 / 2 k} \text { and }
\end{aligned}
$$

$\mathrm{V}=\exp \left\{\mathrm{x}^{2}\left[1-\left(1+4 \mathrm{t}^{2}\right)^{-1 / k}\right]\right\}$. The special case with $\mathrm{m}=0$ is worth noting. Using (3.2) for even case and then applying (2.12) one obtains in a combined form the recurrence relation for the second set 


$$
T_{n}^{\mu}(x ; k)=\sum_{m=0}^{[n / 2]}(-1)^{m} 2^{2 m}(\underset{m}{n / 2})\left(\frac{\mu-\lambda}{k}\right){ }_{m} T_{n-2 m}^{\lambda}(x ; k), \lambda \neq \mu \text { and } \lambda, \mu>-1 / 2 . \text { (3.3) }
$$

Taking $\mu=0$, and $n$ even in (3.3) and using the biorthogonality condition (2.10) we have the integral

$$
\begin{aligned}
& \int_{-\infty}^{\infty}|x|^{2 \lambda} \exp \left(-x^{2}\right) s_{2 m}^{\lambda}(x ; k) T_{2 n}(x ; k) d x \\
= & (-1)^{n} 4^{m+n}(-n)_{m}(-\lambda / k)_{n-m} \Gamma(k m+\lambda+k / 2) \text { where with } \mu=0, T_{2 n}(x ; k) \text { is the }
\end{aligned}
$$

second biorthogonal set suggested by the Hermite polynomials; see Thakare and Madhekar [4]. The integral (3.4) says that $T_{2 n}(x ; k)$ are othogonal to $|x|^{2 \lambda} s_{2 m}^{\lambda}(x ; k)$ w.r.t. the weight function $\exp \left(-x^{2}\right)$ when $n>m+\lambda / k$.

Consider the generating function first given by Genin and Calvez [13]; (see also Karande and Thakare [14], Prabhakar [15]):

$$
\sum_{n=0}^{\infty}(c)_{n} Z_{n}^{\alpha}(x ; k) t^{n} /(1+\alpha)_{k n}=(1-t)^{-c}{ }_{1} F_{k}\left[\begin{array}{l}
c ; \\
\Delta(k, 1+\alpha) ;
\end{array} x^{k} /(1-t) k^{k}\right]
$$

where $|\mathrm{t}|<1$ and $\Delta(\mathrm{m}, \delta)$ stands for the sequence of parameters $\delta / \mathrm{m},(\delta+1) / \mathrm{m}, \ldots$, $(\delta+m-1) / \mathrm{m},(\mathrm{m}>1)$. Using (2.3) one obtains from (3.5), an expression involving even $s_{2 n}^{\mu}(x ; k)$ which after putting to use relation (2.11) gives a corresponding relation for odd $s_{2 n+1}^{\mu}(x ; k)$. This resulting expression further with the help of the relation

$$
\begin{aligned}
& \sum_{n=0}^{\infty}(c) s_{n}^{\mu}{ }_{2 n+1}^{\mu}(x ; k) t^{2 n+1} / n !(\mu+k / 2)_{n k} \\
= & t(k+2 \mu+k \theta) /(k+2 \mu) \sum_{n=0}^{\infty}(c) s_{n}^{\mu}{ }_{2 n+1}^{\mu}(x ; k) t^{2 t} / n !(\mu+1+k / 2)_{n k} \text {, where } \theta=t, d / d t
\end{aligned}
$$

yields

$$
\begin{aligned}
& \sum_{n=0}^{\infty} \frac{(c)_{n}}{n !(\mu+k / 2)_{n k}} s_{2 n+1}^{\mu}(x ; k) t^{2 n+1}=2 t x^{k} U^{-2 k(1+c)}\left(U^{-2 k}-\frac{8 c k t^{2}}{k+2}\right) \cdot \\
& \text { - }{ }_{1} F_{k}\left[\begin{array}{ll}
c ; & W \\
\Delta(k, \mu+1+k / 2) ;
\end{array}\right]+\frac{16 c k t^{3} x^{3 k} U^{2 k(c+2)}}{(k+2 \mu)(1+\mu+k / 2)} \quad 1 F_{k}\left[\begin{array}{ll}
c+1 ; & W \\
\Delta(k, 1+\mu+3 k / 2 ;
\end{array}\right]
\end{aligned}
$$

where $W=4 x^{2 k} t^{2} /\left(1+4 t^{2}\right) k^{k}$.

In fact, one obtains after combining even case with (3.7) the following generating function for the first biorthogonal set $\left\{s_{n}^{\mu}(x ; k)\right\}$ :

$$
\begin{aligned}
& \sum_{n=0}^{\infty} \frac{(c)[n / 2]}{[n / 2] !(\mu+k / 2){ }_{k}[n / 2]} S_{n}^{\mu}(x ; k) t^{n}=\frac{(\mu+k / 2)}{(\mu+1 / 2)} U^{2 k c}{ }_{1} F_{k}\left[\begin{array}{ll}
c ; \\
\Delta(k, \mu+1 / 2) ;
\end{array}\right] \\
& +2 t x^{k} U^{2 k(1+c)}\left(U^{-2 k}-\frac{8 c k t^{2}}{k+2 \mu}\right){ }_{1} F_{k}\left[\begin{array}{ll}
c ; & W(k, 1+\mu+k / 2) ;
\end{array}\right] \\
& +\frac{16 \mathrm{ckt}^{3} x^{3 k} U^{2 k(c+2)}}{(k+3 \mu)(1+\mu+k / 2)}{ }_{k} F_{k}\left[\begin{array}{ll}
c+1 ; & W \\
\Delta(k, 1+\mu+3 k / 2 ;
\end{array}\right] .
\end{aligned}
$$


We finally state the differential equation satisfied by the first set $\left\{S_{n}^{\mu}(x ; k)\right\}$ in the form

$$
\left[x^{2}(x D+2 \mu+1+\varepsilon)\right]^{k}\left\{x^{1-2 k}(D-\varepsilon k / x) s_{n}^{\mu}(x ; k)\right\}
$$

$=\left(2 x^{2}\right)^{k}\left\{x D s_{n}^{\mu}(x ; k)-n k s_{n}^{\mu}(x ; k)\right\}$, and a differential recurrence relation for the second set

$$
k T_{n+2}^{\mu}(x ; k)=-2 x D T_{n}^{\mu}(x ; k)-2\left(1+m 1+2 \mu-2 x^{2}\right) T_{n}^{\mu}(x ; k) .
$$

ACKNOWLEDGEMENTS. The authors are grateful to the referee for fruitful suggestions.

\section{REFERENCES}

1. DIDON, M.F. Sur certains systems des polynômes associes, Annal Sci de l'Ecole Normale Sup 6 (1869), 111-125.

2. DERUYTS, J. Sur Une class de polynômes conjugés, Mem. Cor. et Mem. de Savant Enstr., Acad. Royal des Sci. des letters et des Beaux, Art de Belgique 48 (1886).

3. KONHAUSER, J.D.E. Biorthogonal polynomials suggested by the Laguerre polynomials, Pacific J. Math. 21 (1967), 303-314.

4. THAKARE, N.K. and MADHEKAR, H.C. Biorthogonal polynomials suggested by the Hermite polynomials, Indian J. Pure Appl. Math. 17 (1986), 1031-1041.

5. CHAI, W.A. Problem 72 - 17, "Biorthogonal conditions for a class of polynomials, SIAM Rev. 14 (1972), 494.

6. CARLITZ, L. Problem $72-17$, "Biorthogonal conditions for a class of polynomials, SIAM Rev. 15 (1973), 670-672.

7. MADHEKAR, H.C. and THAKARE, N.K. Biorthogonal polynomials suggested by Jacobi polynomials, Pacific J. Math. 100 (1982), 417-424.

8. SZegö, G. Orthogonal Polynomials, Amer. Math. Soc. Collog. 23 (1974.

9. CARLITZ, L. A Note on certain biorthogonal polynomials, Pacific J. Math. 24 (1968), 425-430.

10. KONHAUSER, J.D.E. Some properties of biorthogonal polynomials, J. Math. Anal. App1. 11 (1965), 242-260.

11. CALVEZ, L.C. and GENIN, R. Appl. des relations entre les fonctions génératrics et les form de type Rodrigues, C.R. Acad. Sci. Paris A-B 270 (1970), A41-A44.

12. SRIVASTAVA, H.M. Some biorthogonal polynomials suggested by the Laguerre polynomials, Pacific J. Math. 98 (1982), 235-250.

13. GENIN, R. and CALVEZ, L.C. Sur les fonctions fe'ne'ratrices de certains polynomes biorthogonaux, C.R. Acad. Sc1. Paris A-B 268 (1969), A1564-A1567.

14. KARANDE, B.K. and THAKARE, N.K. Some results for Konhauser biorthogonal polynomials and dual series equations, Indian J. Pure Appl. Math. 7 (1976), 635-646.

15. PRABHAKAR, T.R. On the other set of biorthogonal polynomials suggested by the Laguerre polynomials, Pacific J. Math. 37 (1971), 801-804.

16. TOSCANO, L. Mathematiche (Catania) 15 (1960) 41-53. 


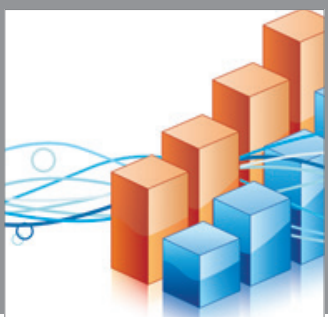

Advances in

Operations Research

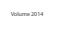

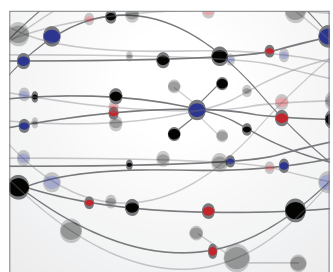

\section{The Scientific} World Journal
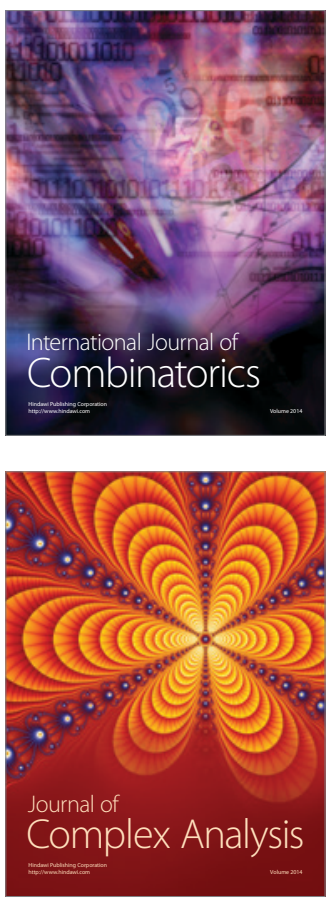

International Journal of

Mathematics and

Mathematical

Sciences
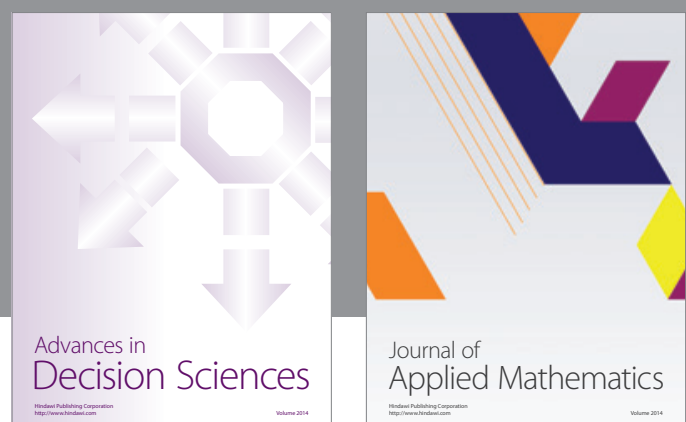

Journal of

Applied Mathematics
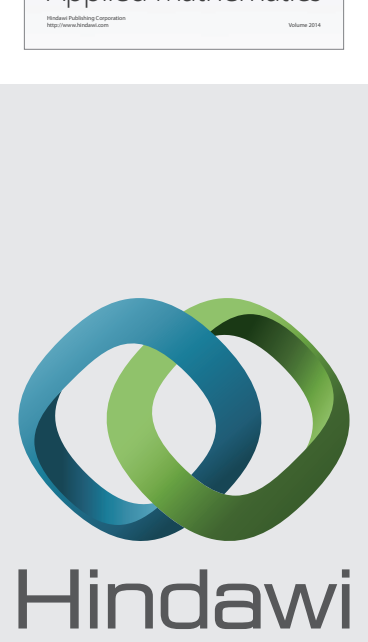

Submit your manuscripts at http://www.hindawi.com
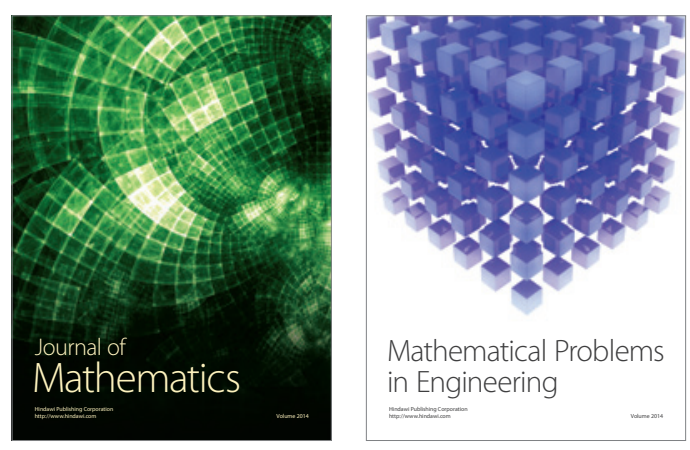

Mathematical Problems in Engineering
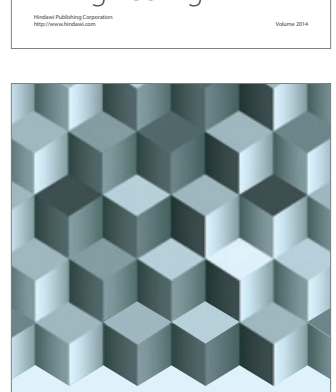

Journal of

Function Spaces
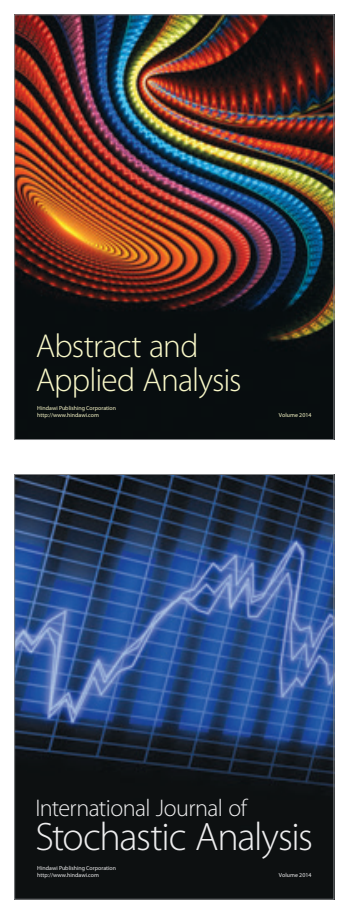

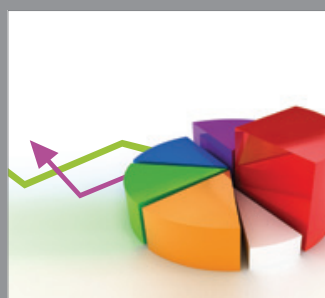

ournal of

Probability and Statistics

Promensencen
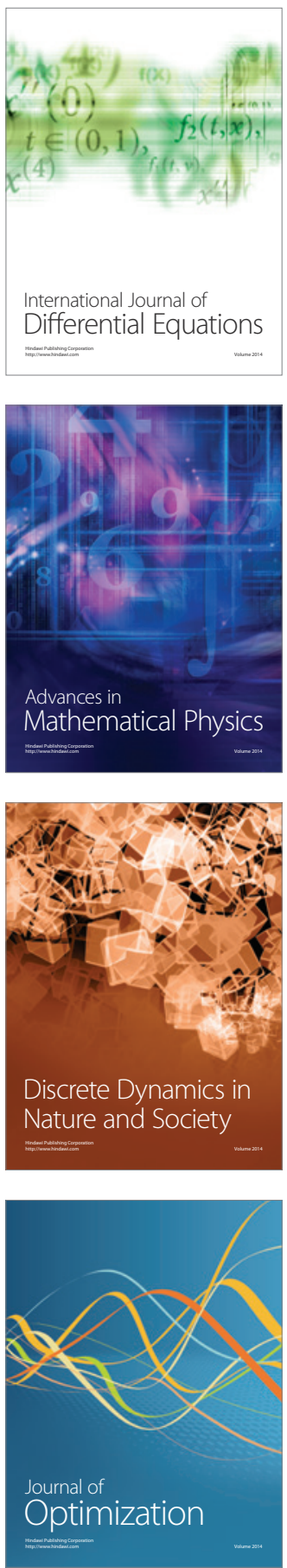